\title{
Caspase recruitment domains for protein interactions in cellular signaling (Review)
}

\author{
HYUN HO PARK \\ Department of Pharmacy, College of Pharmacy, Chung-Ang University, Seoul 06974, Republic of Korea
}

Received August 10, 2018; Accepted January 9, 2019

DOI: $10.3892 / \mathrm{ijmm} .2019 .4060$

\begin{abstract}
The caspase recruitment domain (CARD), a well-known protein interaction module, belongs to the death domain (DD) superfamily, which includes DDs, death effector domains, and pyrin domains. The DD superfamily mediates the protein interactions necessary for apoptosis and immune cell signaling pathways. Among these domains, the CARD has been studied extensively as it mediates important cellular signaling events that are associated with various human diseases including cancer, neuro-degenerative diseases and immune disorders. Homo-type and hetero-type CARD-CARD interactions mediate the formation of large signaling complexes, including caspase-activating complexes and downstream signaling complexes. The present review summarizes and discusses the results of structural studies of various CARDs and their complexes. These studies shed light on the mechanisms that control the assembly and disassembly of signaling complexes and provide an improved understanding of cellular signaling processes.
\end{abstract}

\section{Contents}

1. Introduction

2. Structure of CARDs

3. Structural comparison with other CARDs

4. Dimer interactions of CARDs

5. Oligomeric filament-like assembly of CARDs

6. Future perspectives

\section{Introduction}

A small protein interaction domain composed of $\sim 90$ amino acids, the caspase recruitment domain (CARD) functions in

Correspondence to: Dr Hyun Ho Park, Department of Pharmacy, College of Pharmacy, Chung-Ang University, 84 Heukseok Road, Dongjak, Seoul 06974, Republic of Korea

E-mail: xrayleox@cau.ac.kr

Key words: inflammation, apoptosis, death domain superfamily, caspase-recruitment domain the protein-protein interactions in apoptosis, inflammation and innate cell signaling (1-3). The CARD with the death domain (DD), the death effector domain (DED) and the pyrin domain (PYD) is known as the DD superfamily, which is one of the largest families of protein interaction modules (4-6). The CARD and disruption of CARD-mediated protein interactions have been considered therapeutic targets for cancer (7). In mammals, 33 CARD-containing proteins encoded by various genes have been identified, including several members of the caspase family, and various intracellular signaling proteins that function in the apoptosis, necrosis, innate immunity and inflammation signaling pathways (Fig. 1A) $(5,8)$. The representative CARD-containing proteins in apoptosis signaling are apoptotic protease activating factor-1 (Apaf-1) and receptor-interacting serine/threonine-protein kinase (RIP)-associated protein with a DD (RAIDD) (9-11). They mediate the activation of caspase, and CARDs of Apaf-1 and RAIDDD are critical for the formation of caspase-activating complexes $(12,13)$. Among the two different classes of caspases, the initiator caspases (including caspase-2, -8, -9 and -10) and effector caspases (including caspase-3, -6 and -7), initiator caspases are activated via a proximity-mediated self-activation process. The proximity of initiator caspases is induced by formation of huge molecular complexes (14-16). The apoptosome, which is molecular complex required for the activation of caspase-9, is formed by Apaf-1, which contains a CARD $(17,18)$. The Apaf-1 CARD interacts with the caspase-9 CARD in the formation of an apoptosome $(19,20)$. For the formation of the tumor protein $\mathrm{p} 53$ (p53)-induced protein with a DD (PIDD)osome, which is known as the caspase- 2 activation platform, CARD-containing RAIDD interacts with CARD-containing caspase-2 via a CARD-CARD interaction $(21,22)$. Following DNA damage-induced apoptosis, caspase-2 is recruited to PIDD, which is DD-containing stress indicator, by RAIDD $(9,23)$. In summary, the PIDDosome is formed by DD superfamily mediated interaction of PIDD, RAIDD and caspase-2 $(23,24)$. Similar with apoptosis-associated CARD-containing proteins, several CARD-containing proteins functions in the immune systems. Those include retinoic acid-inducible gene I (RIG-I), RIP2, CARD-containing protein 9 (CARD9), caspase recruitment domain family member 11 (CARMA1), and B-cell lymphoma/leukemia 10 (BCL10) (25). For the proper function of $\mathrm{B}$ and $\mathrm{T}$ cells during innate and adaptive immunity, nuclear factor kappa-light-chain-enhancer of activated $B$ cells (NF-kB) activation via T-cell receptor (TCR) and $\mathrm{B}$-cell receptor (BCR) signaling events are critical. In the 
A

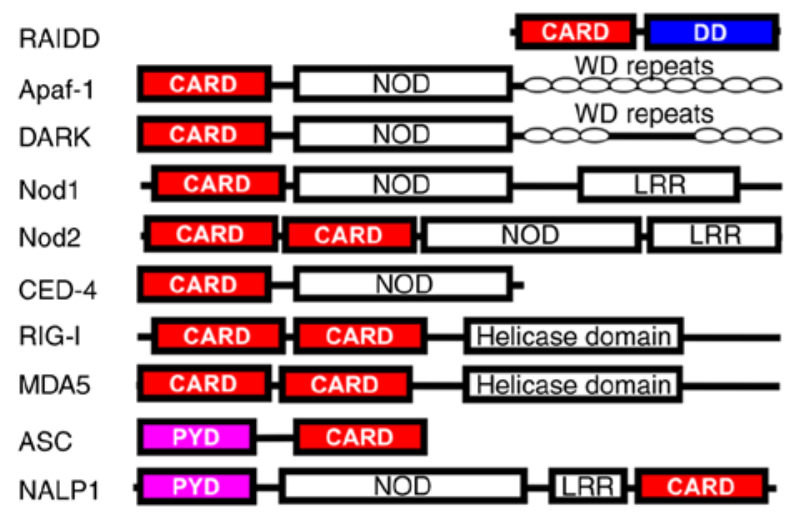

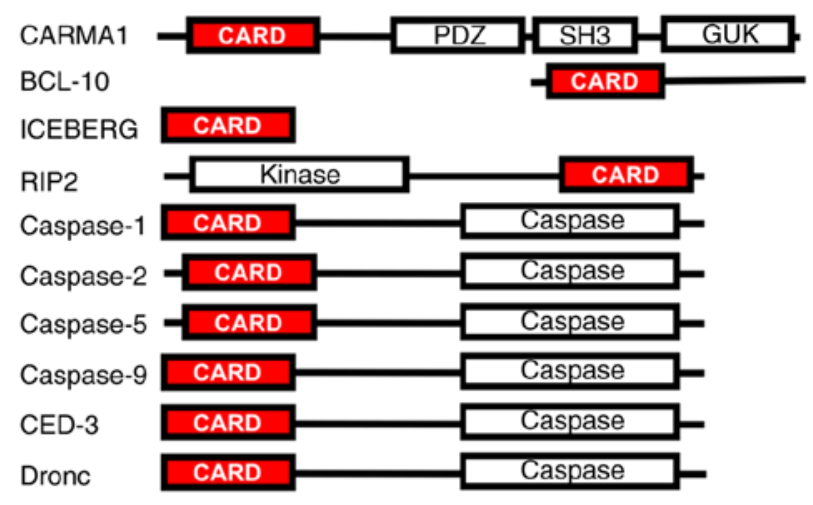

B

\begin{tabular}{|c|c|}
\hline CARMA1 & EEALWDNVECNRHMLSRY I N--PAKL TPYLRQCKV I DEQDEDEVI \\
\hline NALP1 & DAPQLL HFVDQYREQL I ARVT--SVEVVLDKLHGQVLSQE \\
\hline Apaf-1 & MDAKARNCL LQHREA LEKD I K--- TSY I MDHM I SDGFLT- \\
\hline Casp-9 & MDEADRRLLRRCRLRLVEELQ---VDQLWDALLSRE LFRP। \\
\hline NOD1 & ESHPH I QLLKSNREL LVTHI R--NTQCLVDNLLKNDYFSA \\
\hline MAVS & MASDDLSL I RKNRMA LFQQLT--CVLP I LDNLLKANV I NKQEHL \\
\hline ICEBERG & MADQLLRKNRR I F I HSVGAGT I NAL LDCL LEDE V ISQE \\
\hline CARMA1 & LPSKINRAGRLLDI LHTKGQRGYVVFLESLE \\
\hline NALP1 & RPSQMRKLFSLSQSWDRKCKDG---LYQALKETHPHL I ME LWEKG \\
\hline Apaf-1 & EPTQQQRAAML I KM I LKKDNDSY VSFYNALL HEGYKD LAALLHD \\
\hline Casp-9 & SGSRRDQARQL I I DLETRGSQALPLF I SCLEDTGQDM LASFLRTN \\
\hline NOD1 & P-TQPDKVRK I LDLVQSKGEEVSEFFLYLLQQL - ADA YVDLRPWL \\
\hline MAVS & T-QI PLQAREL I DT I L VKGNAAAN I FKNCLKE I DSTL YKNLFVD \\
\hline ICEBERG & D-TVMDKARVL I DLVTGKGPKSCCKF I KHLCEEDPQL AS \\
\hline
\end{tabular}

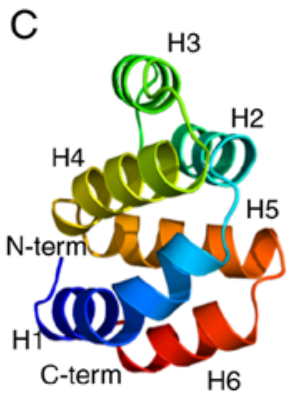

Apaf-1

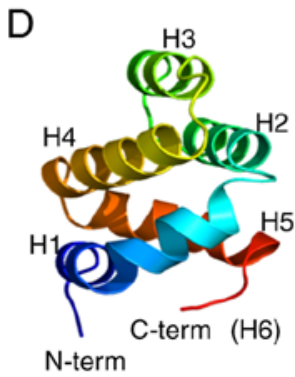

ARC

Figure 1. CARD-containing proteins and the structure of the CARD. (A) Domain boundary of representative CARD-containing proteins. (B) Sequence alignment of selective CARDs. The secondary structures of the CARDs are demonstrated above the corresponding sequence. (C) Representative structure of the CARD formed by 6 helical bundles. Ribbon diagram of Apaf-1 CARD. The chain from the N- to C-termini is colored blue to red. Helices are labeled. (D) Atypical structure of the CARD formed by 5 helical bundles. Ribbon diagram of the ARC CARD. CARD, caspase recruitment domain; DD, death domain; PYD, Pyrin domain; Apaf-1, apoptotic protease activating factor-1; ARC, apoptosis repressor with CARD; DARK, Drosophila Apaf-1-related killer; NOD1/2, nucleotide-binding oligomerization domain-contain protein 1/2; CED-3/4, cell death protein 3/4; RIG-I, retinoic acid-inducible gene I; MDA5, interferon-induced helicase C domain-containing protein 1; ASC, apoptosis-associated speck-like protein containing a CARD; NALP1, NACHT, leucine-rich repeat and PYD domains-containing protein 1; CARMA1, caspase recruitment domain family member 11; Casp-9, caspase 9; MAVS, mitochondrial antiviral-signaling protein; ICEBERG, CARD18; RIP2, receptor-interacting serine/threonine-protein kinase 2; BCL-10, B cell lymphoma/leukemia 10.

early events of TCR and the BCR-mediated NF-kB activation in the immune cells, a CARMA1 signalosome, which is composed of CARD-containing CARMA1, mucosa-associated lymphoid tissue lymphoma translocation protein 1 and an additional CARD-containing BCL10, is formed via CARD-mediated interactions. The scaffolding protein CARMA1, a member of the membrane-associated guanylate kinase family, contains a CARD $(25,26)$. BCL10 also contains a CARD at the $\mathrm{N}$-terminus. The CARD-CARD interaction between CARMA1 and BCL10 is critical for CARMA1 signalosome assembly (27). An additional 
signalosome, the CARD9 signalosome, is the primary signaling molecular complex that performs pivotal roles in immune cells, in particular in NF-kB activation in myeloid cells (28-30). CARD9 is a CARD-containing protein. The structure of the N-terminus of CARD9 is similar to that of CARMA1. For CARD9 signalosome assembly, the adaptor protein BCL10, which contains a CARD at the N-terminus, interacts with CARD9 via a CARD-CARD interaction (30-33). Upon activation of myeloid cells, immunoreceptor-tyrosine-based activation motif-coupled receptors, including dectin-1, triggering receptor expressed on myeloid cells and Fc receptors, which are able to sense danger signals then transfer the signal to form a stable CARD9 signalosome complex, which activates NF- $\kappa \mathrm{B}$ and MAPKs $(28,30)$. CARD-only protein (COPs) have been identified as regulators of innate immunity and inflammation signaling (34). These proteins may inhibit the assembly of nucleotide-binding oligomerization domain (NOD)-like receptor complexes, including the inflammasome and the NODosome, which are critical molecular complexes for caspase-1 activation (35-37). COPs are known to interact with either the caspase-1 CARD or the RIP2 CARD via a CARD-CARD interaction $(38,39)$. A total of 3 COPs, Pseudo interleukin-1 beta converting enzyme, inhibitory CARD and CARD18 (ICEBERG), have been identified in the human genome (34). Several apoptosis inhibitors, including apoptosis repressor with CARD (ARC), also contain CARDs $(40,41)$. ARC may inhibit death-inducing signaling complex assembly followed by caspase activation by binding to the Fas-associated protein with DD (FADD) DD, Fas DD and caspase-8 DED using its CARD (42-44). This protein also inhibits p53 tetramerization and the function of p53 (45). ARC is a molecular target for therapeutic intervention against cancer and hepatocellular necrosis (46-48).

Given that the DD superfamily, in particular the CARD, mediates important cellular signaling pathways that are associated with various human diseases including cancer, neuro-degenerative diseases and immune disorders, studies of CARD-mediated protein interactions and signal transduction are of great biological significance. Structural studies of the CARD and its hetero- and homo-typic complexes have been performed over the previous decade to understand the role of the CARD and how it mediates cellular signaling. As a result, the structures of several CARDs and their complexes have been elucidated, including a recently described filament-like structure (49). The present review summarizes and discusses the current understanding of the structure and function of CARDs in the apoptosis and immune signaling pathways.

\section{Structure of CARDs}

There are two types of CARD-containing proteins: Caspases that contain a CARD as a pro-domain at the $\mathrm{N}$-terminus, and scaffolding adapter proteins required for the assembly of signaling complexes $(1,5)$. At present, the nuclear magnetic resonance (NMR) and crystal structures of 5 and 10 CARD-containing proteins, respectively, representing each of these two types, have been determined. The NMR structures include apoptosis-associated speck-like protein containing a CARD (ASC) CARD (50), BCL10 CARD (27), cellular inhibitor of apoptosis protein 1 CARD (51), RAIDD CARD (24), and ICEBERG (39). Crystal structures are available for Apaf-1 CARD (52,53), BCL10-interacting CARD protein (BinCARD) (54), CARD8 (55), caspase-9 (53), mitochondrial antiviral-signaling protein (MAVS) CARD (56), nucleotide-binding domain, leucine-rich repeat family pyrin domain-containing 1 (NLRP1) CARD (57), NOD-contain protein 1 (NOD1) CARD (58), RIG-I CARD (59), CARD11 (60) and ARC CARD (61). Sequence identity among CARDs is $13-25 \%$ (Fig. 1B).

CARDs adopt a conserved 6-helix bundle fold, which is a common structural feature of the DD superfamily $(2,5)$. Although the CARD exhibits a conserved 6-helix bundle fold, a bent or broken helix $\mathrm{H} 1$ is a unique feature of the CARD structure (8). Notably, the recently elucidated ARC CARD structure exhibited an altered form of the typical DD fold (60). The ARC CARD contains a 5-helix bundle fold (H1-H5) instead of a 6-helix bundle (Fig. 1C and D) $(42,61)$, with helix $\mathrm{H6}$ being disordered and not present in the structure. This indicates that H6 in the CARD is not necessary for its function, and even for the function of the DD superfamily (Fig. 1D). Based on a secondary structure prediction program, the amino acid sequence of the H6 region of the ARC CARD is not predicted to be a typical helix sequence, supporting the current structure, in which $\mathrm{H6}$ does not exist in the ARC CARD and is replaced by a loop instead. This atypical structure of the ARC CARD is the first case of this to be identified among the DD superfamily. Conversely, structural study of the ARC CARD also indicated the typical structural features of a CARD: It contains a short helix $\mathrm{H} 3$, and the helix bundle from $\mathrm{H} 1$ to $\mathrm{H} 5$ is packed by a central hydrophobic core, making the structure compact and stable (61). Compared with other DD superfamily domains, structural rigidity, as a result of the formation of a hydrophobic cluster in the core of the ARC CARD, and a shorter $\mathrm{H} 3$ are common features of the CARD (61). These specific features of the ARC CARD may be critical for the functions of ARC. The association between the missing H6 in the ARC CARD and the capacity of ARC to accommodate various binding partners, including FADD, caspase- 8 and Bcl-2 associated $\mathrm{X}$ protein, should be investigated in future studies.

\section{Structural comparison with other CARDs}

A search of Dali (62), a structural homology search server, indicates that the representative ARC CARD is highly homologous to other CARDs. NOD1, BinCARD, NLRP1, Apaf-1, ICEBERG, CARMA1, RIG-I and RAIDD have been identified as structurally homologous proteins. Clear structural differences, and similarities between ARC CARDs and these other CARDs have been detected by structural comparison using pair-wise structural alignments (Fig. 2A-I). The NOD1 CARD was identified as the being the CARD that may be best superimposed onto the ARC CARD. Notably, the NOD1 CARD also exhibits an atypical structure, in that H6 is associated with $\mathrm{H} 5$ and forms one extended long helix (H5-H6). This atypical H6 helix, which exhibits the dynamic nature of H6, is a noteworthy feature of the ARC and NOD1 CARDs (Fig. 2B). A relatively longer $\mathrm{H} 1$ helix is also identified in the ARC CARD. A structural alignment also exhibited a bent $\mathrm{H} 1$ helix in all of the CARDs, indicating that a bent $\mathrm{H} 1$ helix is a common feature. Although all the CARDs are well 

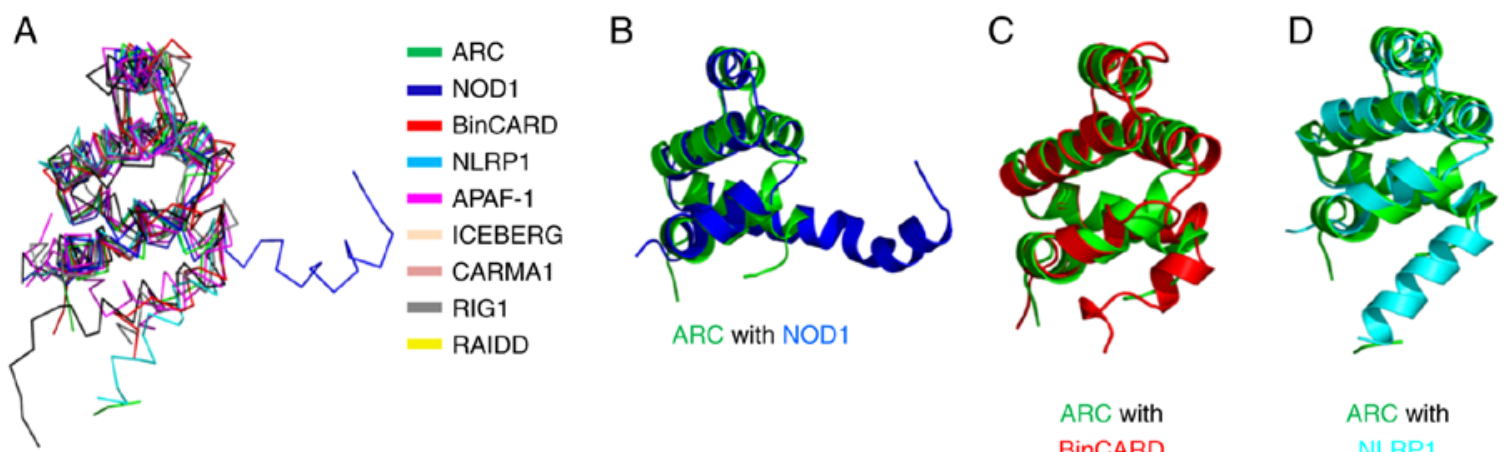

ARC with
BinCARD

ARC with

NLRP1
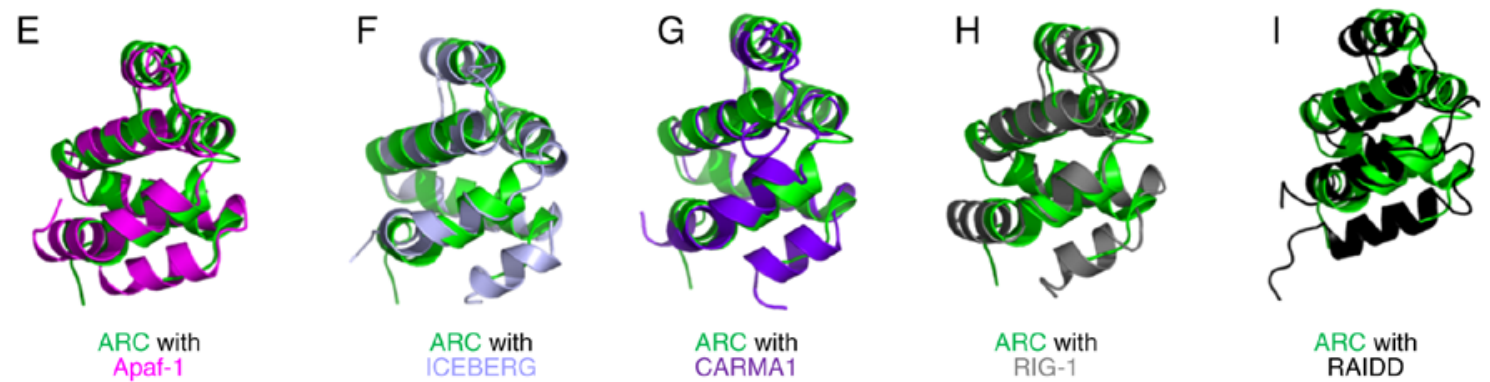

Figure 2. Structural comparison between CARDs. (A) Structures of all CARDs superimposed. Pairwise structural comparisons of (B) NOD1, (C) BinCARD, (D) NLRP1, (E) Apaf-1, (F) ICEBERG, (G) CARMA1, (H) RIG-1 and (I) RAIDD with ARC. CARD, caspase recruitment domain; NOD1, nucleotide-binding oligomerization domain-contain protein 1; BinCARD, B-cell lymphoma/leukemia 10-interacting CARD protein; NLRP1, nucleotide-binding domain, leucine-rich repeat family pyrin domain-containing 1; Apaf1, apoptotic protease activating factor-1; ICEBERG, CARD18; CARMA1, caspase recruitment domain family member 11; RIG-I, retinoic acid-inducible gene I; RAIDD, receptor-interacting serine/threonine-protein kinase-associated protein with a DD; ARC, apoptosis repressor with CARD.

superimposed for $\mathrm{H} 1$ to $\mathrm{H} 5$, superimposition of all 9 CARDs indicated that the lengths and orientations of several helices differ among the different domains (Fig. 2A-I).

\section{Dimer interactions of CARDs.}

The hetero-dimeric structure of a CARD:CARD complex, which indicated the assembly mechanism of the apoptosome required for the activation of caspase- 9 , was provided by the crystal structure of the complex between the Apaf-1 CARD and the caspase-9 CARD (63) (Fig. 3A). This structure was the first structure that exhibited the protein interaction interface of a DD superfamily member. The structure of the CARD complex indicated that there was a mutual recognition between the concave surface of the caspase-9 CARD and the convex surface of the Apaf-1 CARD. The primary force for the interaction is driven by a massive number of salt bridges formed between the positively charged $\mathrm{H} 1$ and $\mathrm{H} 4$ helices in the caspase-9 domain and the negatively charged $\mathrm{H} 2$ and $\mathrm{H} 3$ helices in the Apaf- 1 domain. A total of 3 positively charged residues, R13, R52 and R56 in the caspase-9 domain interact with 2 negatively charged residues, D27 and E40. This ionic nature of the Apaf-1 CARD: Caspase-9 CARD interaction was considered to be the typical DD superfamily interaction, until the new structure of a DD complex was elucidated: Park et al (64) demonstrated the first structure of a highly oligomeric DD complex, which introduced 3 novel types of interactions (types I, II, and III) at 6 unique interfaces (types Ia, Ib, IIa, IIb, IIIa, and IIIb; Fig. 3B) (65). Based on this structure, it was then identified that the caspase-9 CARD/Apaf-1 CARD complex is formed by a type I interaction (63). The involvement of the $\mathrm{H} 1$ and $\mathrm{H} 4$ helices in the caspase-9 CARD, a typical Type Ia surface, and $\mathrm{H} 2$ and $\mathrm{H} 3$ helices in the Apaf-1 CARD, a typical Type Ib surface, supports the hypothesis that the caspase- 9 CARD/Apaf-1 CARD complex is formed by a type I interaction. Unlike DDs and DEDs, for which there have been several studies, it is unclear whether CARDs may also self-associate. In a full-length structural study using electron microscopy, the Apaf-1 CARD appeared to form a really interesting new gene-complex structure near the center of the apoptosome, with or without full-length caspase-9 (66). However, monomeric forms of the Apaf-1 CARD have also been described, indicating that CARDs, or at least those present with an oligomerization NOD and those in the pro-domains of caspases, do not possess the ability to stably self-associate, and are instead primarily involved in the interaction with other CARDs. Jang et al (61) introduced the first homo-dimeric structure of the CARD in 2015, with a structural study of the ARC CARD. Prior to this structural study, dimerization of ARC via the CARD had been suggested (43). The anti-apoptotic function of ARC is abolished by CARD-mediated dimerization. Although CARD dimerization had been suggested in a number of studies, this ARC CARD structure was the first structure that demonstrated direct evidence of a homo-dimeric CARD interaction $(4,60,65)$. The interaction mode of the ARC homo-dimer was almost identical with that of the hetero-dimeric complex formed between the caspase-9 and Apaf-1 CARDs. Positively charged amino acid residues from $\mathrm{H} 1$ and $\mathrm{H} 4$ of one ARC CARD molecule form charge-charge interactions with negatively charged $\mathrm{H} 2$ and $\mathrm{H} 3$ in the other CARD molecule (63). Among the 3 different types of interactions detected in the DD hetero complex between the RAIDD DD domain and the PIDD DD domain, this mode of 
A

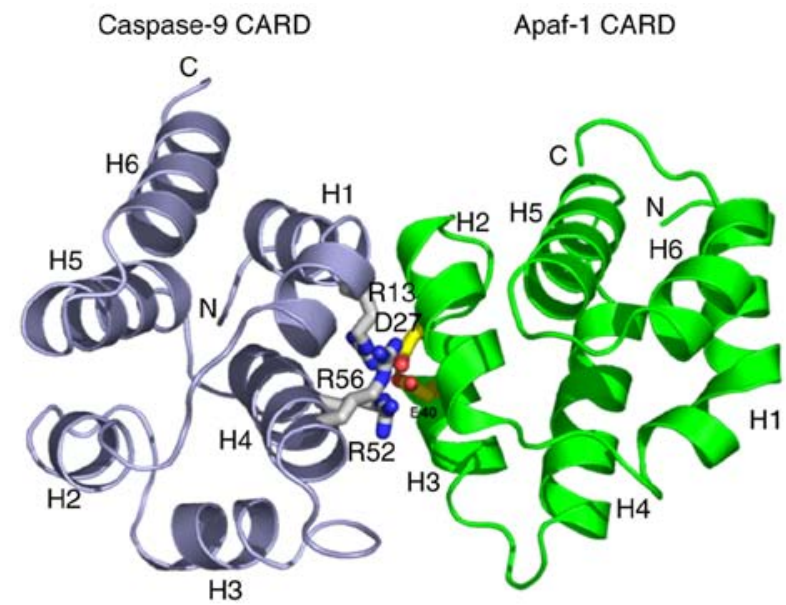

B

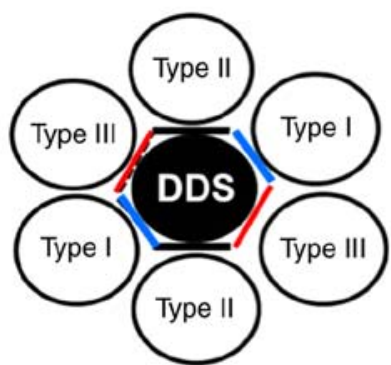

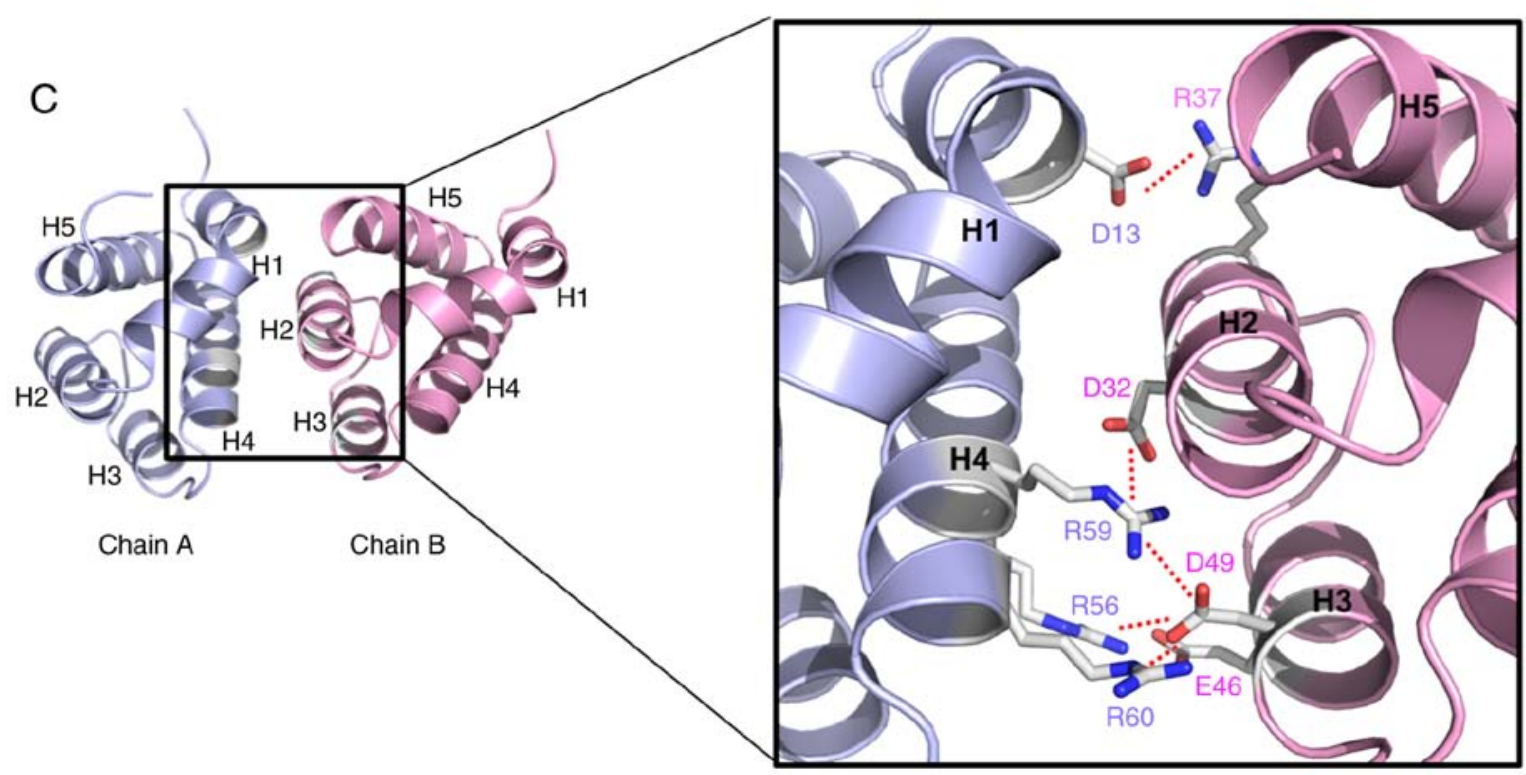

Figure 3. Typical features of dimeric interface of the CARD. (A) Prototype hetero-dimeric interface of CARD-CARD interaction that was introduced by the structural study of the complex formed by the caspase-9 and Apaf-1 CARDs. (B) Potential interaction types formed by the DDS. (C) The prototype homo-dimeric interface of CARD-CARD interaction that was introduced by the structural study of homo-dimeric structure of the ARC CARD. The details of interface are visualized in close detail. Interface residues that are involved in the formation of interface are represented by sticks and the massive salt bridges formed are represented by red dashed lines. CARD, caspase recruitment domain; Apaf-1, apoptotic protease activating factor-1; DDS, death domain superfamily; ARC, apoptosis repressor with CARD.

interaction is classified as a type I interaction $(6,64)$. A total of 2 asymmetric ARC CARD dimers were detected in the asymmetric unit (Fig. 3C). Residues D13, R56, R59 and R60 from one ARC CARD molecule form massive salt bridges and hydrogen bonds with residues D32, R37, E46 and D49 from the other ARC CARD molecule (Fig. 3C). An additional molecular basis for homo-dimerization of CARD was revealed by structural studies of the CARMA1 CARD interface (60). Specifically, unlike the ARC CARD, 2 CARMA1 CARDs in the asymmetric unit form a symmetric dimer. Notably, a limited number of interaction forces are present at the interaction interface. The primary interaction forces for this homo-dimer of the CARD were an electrostatic interaction between residue H31 from one CARMA1 CARD and residue E27 from the other CARMA1 CARD, and a disulfide-bond formed by $\mathrm{C} 28$ in each molecule. The H1 helices in the 2 CARMA1 CARDs were linked by this disulfide bond (Fig. 4). This was the first study that demonstrated disulfide bond-mediated homo-dimerization of a CARD (60).

\section{Oligomeric filament-like assembly of CARDs}

A notable oligomerization mechanism in hetero CARD complexes was recently revealed by a structural study of the RIG-I and MAVS complex $(49,67)$. This structure was the first to exhibit a helical assembly of the CARD, similar to that of the previously solved structure of the DD complex. In this study, 2 oligomeric forms of the CARD were demonstrated, a homo-tetramer and a filament-like structure formation (67). The tandem CARDs in RIG-I form a tetramer in solution, leading to the MAVS CARD interaction and filament formation (Fig. 5A). A total of 3 known types of interactions, namely Ia:Ib, IIa:IIb, and IIIa:IIIb, mediate the assembly of the CARD oligomer. This structural study clearly demonstrated that the surfaces of one MAVS CARD interact with the surfaces of the CARD2 domain in the RIG-I tandem CARDs complex, using all the interaction types that have been identified for the DD complex, namely type I, type II, and type III. Based on the structure of the RIG-I tandem CARDs and the MAVS 


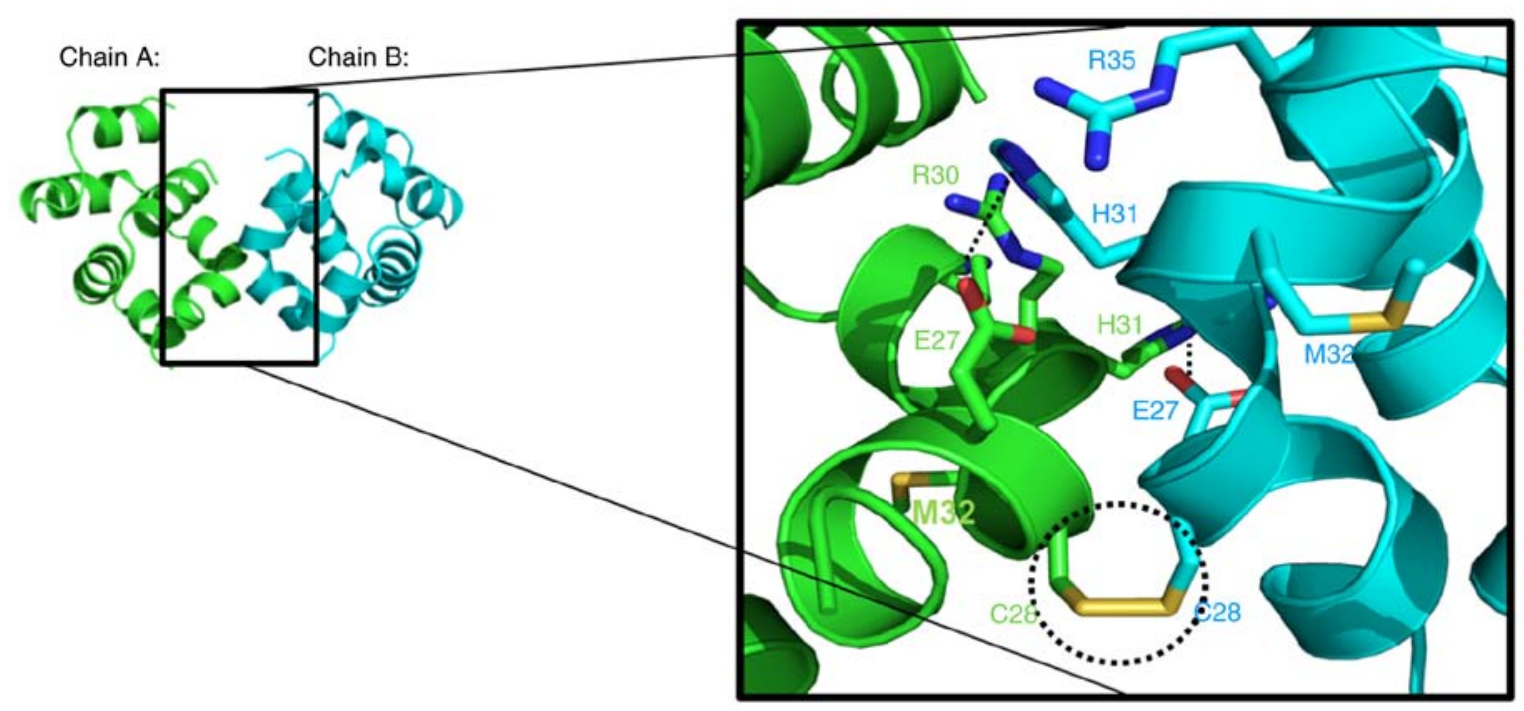

Figure 4. Unusual homo-dimeric structure of CARD. Atypical homo-dimeric interaction was introduced at the structural study of CARMA1 CARD. The details of interface are visualized in close detail. The magnified box demonstrates the residues involved in the contact. CARD, caspase recruitment domain; CARMA1, caspase recruitment domain family member 11.

CARDs complex, the authors suggested a 'lock-washer model', in which RIG-I forms a tandem CARD tetramer, termed the 'lock-washer'; this causes the formation of a MAVS CARD filament, which triggers downstream signaling. Although several studies have suggested that CARD-containing proteins may form filament-like structures through CARD-CARD interactions $(27,68)$, this structure was the first to exhibit the filament-like structures of the CARD (66). From the structural study of the MAVS CARD, it was identified that 1 CARD interacts with 2 neighboring MAVS CARDs via type I, II, and III interactions. The filament-like structure formed by the MAVS CARD consists of a left-handed single-strand helix (Fig. 5B and C) (49). Electrostatic and hydrophobic interactions were identified in the homo-dimeric interactions within a filament (49). Amino acid residues R37, D40, R41, A44 and L48 in one MAVS CARD interact with residues R52, D53, G50 and L48 in a second MAVS CARD, forming a typical type III interaction. Amino acid residues R43 and W56 are involved in the type I interaction. Amino acid residues R37, R41, R65 and R64 in one MAVS CARD and N21, D23 and E26 in a second MAVS CARD are involved in the formation of the type II interaction. This type of helical and filament-like structure was first identified in a structural study of the DD complex, including the PIDDosome $(5,64)$ (Fig. 5D and $\mathrm{E})$.

\section{Future perspectives}

In humans, $\sim 33$ CARD-containing proteins have been identified. Due to the importance of their role in various signaling pathways, these CARD-containing proteins, and the CARD itself, have been intensively studied. The CARD is the second largest subfamily among the DD superfamily which includes DD, DED and PYD. Homo-type and hetero-type CARD-CARD interactions mediate the formation of huge signaling complexes, including caspase activating complexes and downstream signaling complexes, during the cellular signaling process. Understanding of the structures and func- tions of CARD-containing proteins has progressed rapidly. Based on their structures and functions, CARD-containing proteins may be subdivided into two subgroups; those with a pro-domain in the caspase, and those in downstream signaling molecules. Although all CARDs share a low sequence identity, CARDs adopt the typical structural features of the DD superfamily, with a 6-helix bundle. The only distinct feature of the CARD is that helix $\mathrm{H} 1$ tends to bend, or be broken into separate H1a and H1b helices. An additional feature of the CARD is the formation of a core hydrophobic cluster that connects all 6 helices. Recently, an atypical CARD structure was revealed from the structural study of the ARC CARD (61). Typical homotypic interactions between CARDs occur through charge-charge interactions, which was first demonstrated by the structural study of the caspase-9/Apaf-1 complex (63). To understand the relevance of the mechanism of CARD-mediated complex formation and downstream signaling pathways, it is important to investigate the oligomerization mechanism of CARD-containing proteins. CARD dimerization is one simple oligomerization method and may involve hetero- and homo-dimerization. For example, Apaf-1 and caspase- 9 form a hetero-dimeric structure through a type I interface. A total of 2 different methods of homo-dimerization have recently been identified from the structural studies of the CARMA1 and ARC CARDs $(60,61)$. Specifically, the CARMA1 CARD dimerizes through an intermolecular disulfide bond, which has not been observed in any other member of the DD superfamily. This novel method of oligomerization requires additional study in more detail to understand the role of disulfide-bond mediated dimerization in intracellular signaling pathways. The interaction mode of the ARC homo-dimer is similar to that of the hetero-dimeric complex formed between the caspase-9 and Apaf-1 CARDs. In each of these cases, helices $\mathrm{H} 1$ and $\mathrm{H} 4$ in one CARD molecule interact with helices $\mathrm{H} 2$ and $\mathrm{H} 3$ in a second CARD molecule, primarily via charge-charge interactions.

A recent structural study of the RIG-I and MAVS complex suggests that the CARDs in RIG-I may form a tetramer that 
A

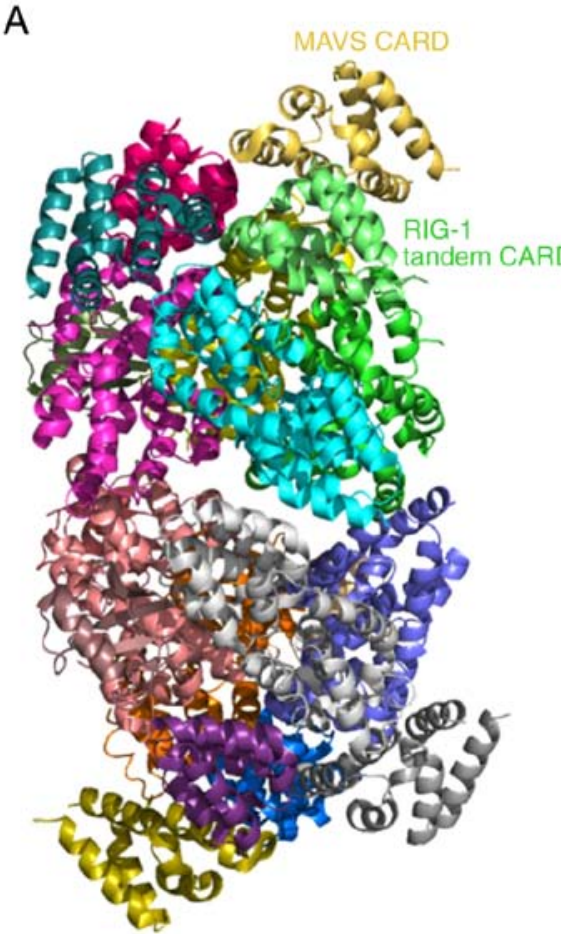

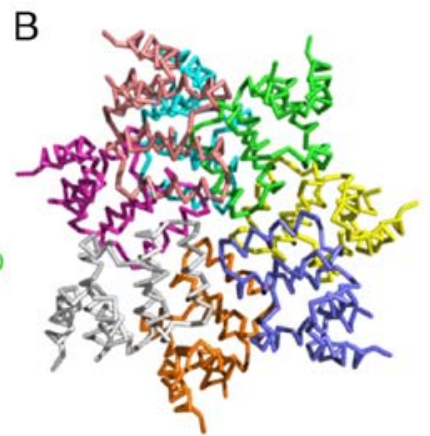

D

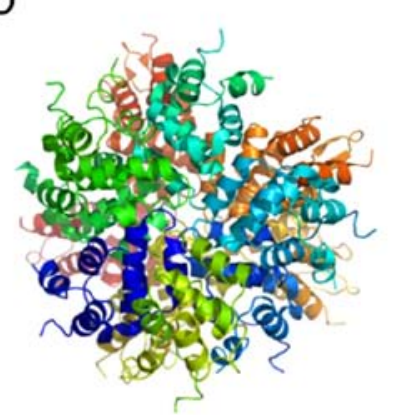

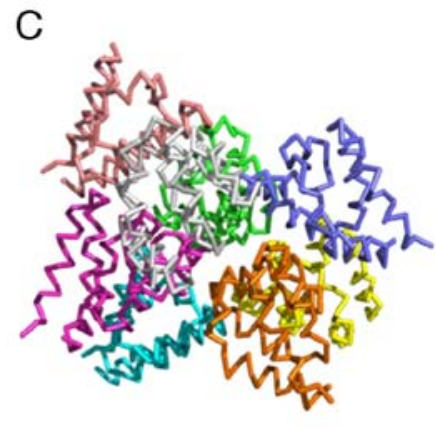

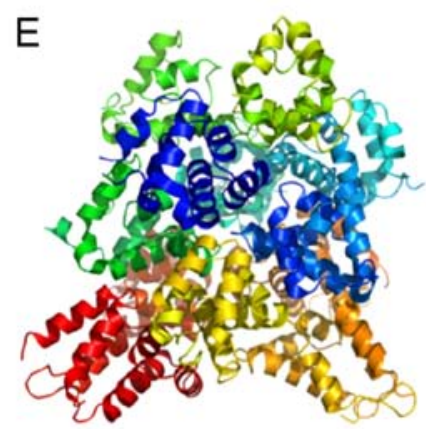

Figure 5. Helical filament-like assembly of CARDs. (A) Helical assembly of RIG-I CARD and MAVS CARD. The tandem CARD of RIG-I that formed tetramer functions as a scaffold for MAVS assembly. (B) Top and (C) side view of the helical assembly of the MAVS CARD. Filament-like assembly was revealed by the structural study of MAVS CARDs. The left-handed helix filament-like assembly was formed by 3 typical types of DD superfamily interactions. (D) Top and (E) side view of the first helical assembly of the DD superfamily, which was introduced by the structural study of hetero oligomeric DD complex of RAIDD DD and PIDD DD. CARDs, caspase recruitment domain; RIG-1, retinoic acid-inducible gene I; MAVS, mitochondrial antiviral-signaling protein; DD, death domain; RAIDD, receptor-interacting serine/threonine-protein kinase-associated protein with a DD; PIDD, tumor protein 553 -induced protein with a DD.

generates a stage for the MAVS CARD to build a left-handed single-strand helical filament (67). The interaction between the first and second CARDs in RIG-I has been demonstrated to stabilize the tandem CARD tetramer $(59,67)$. This structure of the MAVS CARD and the tandem RIG-1 CARD complex indicated that all three types of interactions, namely type I, type II and type III are used for filament assembly, and the locations of the type I, II and III surfaces on the CARD are similar to those on the DD complex. Additionally, surface and charge complementarity have been indicated to be essential for binding specificity in PIDDosome formation. The features of a CARD surface including acidic, basic and hydrophobic patches, in addition to the shape of the surface may therefore be important for specific CARD-CARD interactions. Homo-filament structures have also been demonstrated using cryo-electron microscopy structural studies of caspase-1, ASC and NLRC4 CARDs (69,70). These studies demonstrated the formation of a canonical helical filament assembly using typical type I, type II and type III interactions.

The elucidation of the mechanisms that control the assembly and disassembly of the signaling complexes is important to gain an understanding of cellular signaling processes. The present review highlights the versatile roles of CARDs, the limited structures of CARD complexes, and the dimeric or helical assembly of CARDs. Whether other CARD-containing signaling complexes utilize similar mechanisms for the assembly of complexes is an important topic for future study.

\section{Acknowledgements}

The author would like to thank Mr. Chang Min Kim for assisting with the production of figures.

\section{Funding}

The present study was supported by the Basic Science Research Program through the National Research Foundation of Korea of the Ministry of Education, Science and Technology (grant nos. NRF-2017M3A9D8062960 and NRF-2018R1A2B2003635) and a grant from the Korea Healthcare Technology R\&D Project, Ministry of Health and Welfare, Republic of Korea (grant no. HI17C0155).

\section{Availability of data and materials}

All data generated or analyzed during this study are included in this published article.

\section{Authors' contributions}

HHP conceived and designed the study, performed the literature search and wrote the manuscript.

\section{Ethics approval and consent to participate}

Not applicable. 


\section{Patient consent for publication}

Not applicable.

\section{Competing interests}

The author declares no competing interests in this study.

\section{References}

1. Bouchier-Hayes L and Martin SJ: CARD games in apoptosis and immunity. EMBO Rep 3: 616-621, 2002.

2. Hofmann K, Bucher P and Tschopp J: The CARD domain: A new apoptotic signalling motif. Trends Biochem Sci 22: 155-156, 1997.

3. Kao WP, Yang CY, Su TW, Wang YT, Lo YC and Lin SC: The versatile roles of CARDs in regulating apoptosis, inflammation, and NF- $\mathrm{kB}$ signaling. Apoptosis 20: 174-195, 2015.

4. Bae JY and Park HH: Crystal structure of NALP3 protein pyrin domain (PYD) and its implications in inflammasome assembly. J Biol Chem 286: 39528-39536, 2011.

5. Park HH, Lo YC, Lin SC, Wang L, Yang JK and Wu H: The death domain superfamily in intracellular signaling of apoptosis and inflammation. Ann Rev Immunol 25: 561-586, 2007.

6. Park HH: Structural analyses of death domains and their interactions. Apoptosis 16: 209-220, 2011.

7. Damiano JS and Reed JC: CARD proteins as therapeutic targets in cancer. Curr Drug Targets 5: 367-374, 2004

8. Kwon D, Yoon JH, Shin SY, Jang TH, Kim HG, So I, Jeon JH and Park HH: A comprehensive manually curated protein-protein interaction database for the Death Domain superfamily. Nucleic Acids Res 40: D331-D336, 2012.

9. Duan H and Dixit VM: RAIDD is a new 'death' adaptor molecule. Nature 385: 86-89, 1997.

10. Rodriguez J and Lazebnik Y: Caspase-9 and APAF-1 form an active holoenzyme. Genes Dev 13: 3179-3184, 1999.

11. Ahmad M, Srinivasula SM, Wang L, Talanian RV, Litwack G, Fernandes-Alnemri T and Alnemri ES: CRADD, a novel human apoptotic adaptor molecule for caspase-2, and FasL/tumor necrosis factor receptor-interacting protein RIP. Cancer Res 57: 615-619, 1997.

12. Denault JB and Salvesen GS: Caspases: Keys in the ignition of cell death. Chem Rev 102: 4489-4500, 2002.

13. Donepudi M and Grutter MG: Structure and zymogen activation of caspases. Biophys Chem 101-102: 145-153, 2002.

14. Ludwig-Galezowska AH, Flanagan L and Rehm M: Apoptosis repressor with caspase recruitment domain, a multifunctional modulator of cell death. J Cell Mol Med 15: 1044-1053, 2011.

15. Salvesen GS: Caspases and apoptosis. Essays Biochem 38: 9-19, 2002.

16. Salvesen GS and Dixit VM: Caspases: Intracellular signaling by proteolysis. Cell 91: 443-446, 1997.

17. Adams JM and Cory S: Apoptosomes: Engines for caspase activation. Curr Opin Cell Biol 14: 715-720, 2002.

18. Pop C, Timmer J, Sperandio S and Salvesen GS: The apoptosome activates caspase-9 by dimerization. Mol Cell 22: 269-275, 2006.

19. Acehan D, Jiang X, Morgan DG, Heuser JE, Wang X and Akey CW: Three-dimensional structure of the apoptosome: Implications for assembly, procaspase-9 binding, and activation. Mol Cell 9: 423-432, 2002.

20. Yu X, Acehan D, Menetret JF, Booth CR, Ludtke SJ, Riedl SJ, Shi Y, Wang X and Akey CW: A structure of the human apoptosome at $12.8 \mathrm{~A}$ resolution provides insights into this cell death platform. Structure 13: 1725-1735, 2005.

21. Park HH: Structural features of caspase-activating complexes. Int J Mol Sci 13: 4807-4818, 2012.

22. Manzl C, Krumschnabel G, Bock F, Sohm B, Labi V Baumgartner F, Logette E, Tschopp J and Villunger A: Caspase-2 activation in the absence of PIDDosome formation. J Cell Biol 185: 291-303, 2009.

23. Tinel A and Tschopp J: The PIDDosome, a protein complex implicated in activation of caspase-2 in response to genotoxic stress. Science 304: 843-846, 2004.

24. Chou JJ, Matsuo H, Duan H and Wagner G: Solution structure of the RAIDD CARD and model for CARD/CARD interaction in caspase-2 and caspase-9 recruitment. Cell 94: 171-180, 1998.
25. Gaide O, Martinon F, Micheau O, Bonnet D, Thome M and Tschopp J: Carma1, a CARD-containing binding partner of Bcl10, induces Bcl10 phosphorylation and NF-kappaB activation. FEBS Lett 496: 121-127, 2001

26. Bertin J, Wang L, Guo Y, Jacobson MD, Poyet JL, Srinivasula SM, Merriam S, DiStefano PS and Alnemri ES: CARD11 and CARD14 are novel caspase recruitment domain (CARD)/membrane-associated guanylate kinase (MAGUK) family members that interact with BCL10 and activate NF-kappa B. J Biol Chem 276: 11877-11882, 2001.

27. Qiao Q, Yang C, Zheng C, Fontan L, David L, Yu X, Bracken C, Rosen M,Melnick A,Egelman EHandWuH: Structural architecture of the CARMA1/Bcl10/MALT1 signalosome: Nucleation-induced filamentous assembly. Mol Cell 51: 766-779, 2013.

28. Hara H, Ishihara C, Takeuchi A, Imanishi T, Xue L, Morris SW, Inui M, Takai T, Shibuya A, Saijo S, et al: The adaptor protein CARD9 is essential for the activation of myeloid cells through ITAM-associated and Toll-like receptors. Nat Immunol 8: 619-629, 2007.

29. Hsu YM, Zhang Y, You Y, Wang D, Li H, Duramad O, Qin XF, Dong $\mathrm{C}$ and Lin $\mathrm{X}$ : The adaptor protein CARD9 is required for innate immune responses to intracellular pathogens. Nat Immunol 8: 198-205, 2007.

30. Hara $\mathrm{H}$ and Saito T: CARD9 versus CARMA1 in innate and adaptive immunity. Trends Immunol 30: 234-242, 2009.

31. Uren AG, O'Rourke K, Aravind LA, Pisabarro MT, Seshagiri S, Koonin EV and Dixit VM: Identification of paracaspases and metacaspases: Two ancient families of caspase-like proteins, one of which plays a key role in MALT lymphoma. Mol Cell 6: 961-967, 2000.

32. Lucas PC, Yonezumi M, Inohara N, McAllister-Lucas LM, Abazeed ME, Chen FF, Yamaoka S, Seto M and Nunez G: Bcl10 and MALT1, independent targets of chromosomal translocation in malt lymphoma, cooperate in a novel NF-kappa B signaling pathway. J Biol Chem 276: 19012-19019, 2001.

33. Thome M: CARMA1, BCL-10 and MALT1 in lymphocyte development and activation. Nat Rev Immunol 4: 348-359, 2004.

34. Le HT and Harton JA: Pyrin- and CARD-only proteins as regulators of NLR functions. Front Immunol 4: 275, 2013.

35. Martinon F, Burns K and Tschopp J: The inflammasome: A molecular platform triggering activation of inflammatory caspases and processing of proIL-beta. Mol Cell 10: 417-426, 2002.

36. Stehlik C and Dorfleutner A: COPs and POPs: Modulators of inflammasome activity. J Immunol 179: 7993-7998, 2007.

37. Franchi L, Eigenbrod T, Munoz-Planillo R and Nunez G: The inflammasome: A caspase-1-activation platform that regulates immune responses and disease pathogenesis. Nat Immunol 10: 241-247, 2009.

38. Lee SH, Stehlik C and Reed JC: Cop, a caspase recruitment domain-containing protein and inhibitor of caspase-1 activation processing. J Biol Chem 276: 34495-34500, 2001.

39. Humke EW, Shriver SK, Starovasnik MA, Fairbrother WJ and Dixit VM: ICEBERG: A novel inhibitor of interleukin-1beta generation. Cell 103: 99-111, 2000

40. Abmayr S, Crawford RW and Chamberlain JS: Characterization of ARC, apoptosis repressor interacting with CARD, in normal and dystrophin-deficient skeletal muscle. Hum Mol Genet 13: 213-221, 2004

41. Koseki T, Inohara N, Chen S and Nunez G: ARC, an inhibitor of apoptosis expressed in skeletal muscle and heart that interacts selectively with caspases. Proc Natl Acad Sci USA 95: 5156-5160, 1998.

42. Ha HJ and Park HH: Molecular basis for the effect of the L31F mutation on CARD function in ARC. FEBS Lett 591: 2919-2928, 2017.

43. Nam YJ, Mani K, Ashton AW, Peng CF, Krishnamurthy B, Hayakawa Y, Lee P, Korsmeyer SJ and Kitsis RN: Inhibition of both the extrinsic and intrinsic death pathways through nonhomotypic death-fold interactions. Mol Cell 15: 901-912, 2004.

44. Park HH: Molecular basis of dimerization of initiator caspase was revealed by crystal structure of caspase- 8 pro-domain. Cell Death Differ 11 Sep, 2018.

45. Foo RS, Nam YJ, Ostreicher MJ, Metzl MD, Whelan RS, Peng CF Ashton AW, Fu W, Mani K, Chin SF, et al: Regulation of p53 tetramerization and nuclear export by ARC. Proc Natl Acad Sci USA 104: 20826-20831, 2007.

46. An J,Mehrhof F,Harms C,Lattig-Tunnemann G,Lee SL,Endres M, Li M, Sellge G, Mandic AD, Trautwein C and Donath S: ARC is a novel therapeutic approach against acetaminophen-induced hepatocellular necrosis. J Hepatol 58: 297-305, 2013. 
47. Mercier I, Vuolo M, Jasmin JF, Medina CM, Williams M, Mariadason JM, Qian H, Xue X, Pestell RG, Lisanti MP and Kitsis RN: ARC (apoptosis repressor with caspase recruitment domain) is a novel marker of human colon cancer. Cell Cycle 7: 1640-1647, 2008.

48. Mercier I, Vuolo M, Madan R, Xue X, Levalley AJ, Ashton AW, Jasmin JF, Czaja MT, Lin EY, Armstrong RC, et al: ARC, an apoptosis suppressor limited to terminally differentiated cells, is induced in human breast cancer and confers chemo- and radiation-resistance. Cell Death Differ 12: 682-686, 2005.

49. Xu H, He X, Zheng H, Huang LJ, Hou F, Yu Z, de la Cruz MJ, Borkowski B, Zhang X, Chen ZJ and Jiang QX: Structural basis for the prion-like MAVS filaments in antiviral innate immunity. Elife 3: e01489, 2014

50. de Alba E: Structure and interdomain dynamics of apoptosis-associated speck-like protein containing a CARD (ASC). J Biol Chem 284: 32932-32941, 2009.

51. Lopez J, John SW, Tenev T, Rautureau GJ, Hinds MG, Francalanci F, Wilson R, Broemer M, Santoro MM, Day CL and Meier P: CARD-mediated autoinhibition of cIAP1's E3 ligase activity suppresses cell proliferation and migration. Mol Cell 42: 569-583, 2011.

52. Shiozaki EN, Chai J and Shi Y: Oligomerization and activation of caspase-9, induced by apaf-1 CARD. Proc Natl Acad Sci USA 99: 4197-4202, 2002.

53. Zhou P, Chou J, Olea RS, Yuan J and Wagner G: Solution structure of Apaf-1 CARD and its interaction with caspase-9 CARD: A structural basis for specific adaptor/caspase interaction. Proc Natl Acad Sci USA 96: 11265-11270, 1999.

54. Chen KE, Richards AA, Caradoc-Davies TT, Vajjhala PR, Robin G, Lua LH, Hill JM, Schroder K, Sweet MJ, Kellie S, et al: The structure of the caspase recruitment domain of BinCARD reveals that all three cysteines can be oxidized. Acta Crystallogr D Biol Crystallogr 69: 774-784, 2013

55. Jin T, Huang M, Smith P, Jiang J and Xiao TS: The structure of the CARD8 caspase-recruitment domain suggests its association with the FIIND domain and procaspases through adjacent surfaces. Acta Crystallogr Sect F Struct Biol Cryst Commun 69: 482-487, 2013.

56. Potter JA, Randall RE and Taylor GL: Crystal structure of human IPS-1/MAVS/VISA/Cardif caspase activation recruitment domain. BMC Struct Biol 8: 11, 2008.

57. Jin T, Curry J, Smith P, Jiang J and Xiao TS: Structure of the NLRP1 caspase recruitment domain suggests potential mechanisms for its association with procaspase-1. Proteins 81: 1266-1270, 2013.

58. Manon F, Favier A, Nunez G, Simorre JP and Cusack S: Solution structure of NOD1 CARD and mutational analysis of its interaction with the CARD of downstream kinase RICK. J Mol Biol 365: 160-174, 2007.
59. Ferrage F, Dutta K, Nistal-Villan E,Patel JR, Sanchez-Aparicio MT, De Ioannes P, Buku A, Aseguinolaza GG, Garcia-Sastre A and Aggarwal AK: Structure and dynamics of the second CARD of human RIG-I provide mechanistic insights into regulation of RIG-I activation. Structure 20: 2048-2061, 2012.

60. Jang TH, Park JH and Park HH: Novel disulfide bond-mediated dimerization of the CARD domain was revealed by the crystal structure of CARMA1 CARD. PLoS One 8: e79778, 2013.

61. Jang TH, Kim SH, Jeong JH, Kim S, Kim YG and Park HH: Crystal structure of caspase recruiting domain (CARD) of apoptosis repressor with CARD (ARC) and its implication in inhibition of apoptosis. Sci Rep 5: 9847, 2015.

62. Holm L and Sander C: Dali: A network tool for protein structure comparison. Trends Biochem Sci 20: 478-480, 1995.

63. Qin H, Srinivasula SM, Wu G, Fernandes-Alnemri T, Alnemri ES and Shi Y: Structural basis of procaspase-9 recruitment by the apoptotic protease-activating factor 1. Nature 399: 549-557, 1999.

64. Park HH, Logette E, Rauser S, Cuenin S, Walz T, Tschopp J and Wu H: Death domain assembly mechanism revealed by crystal structure of the oligomeric PIDDosome core complex. Cell 128: 533-546, 2007.

65. Coussens NP, Mowers JC, McDonald C, Nunez G and Ramaswamy S: Crystal structure of the Nod1 caspase activation and recruitment domain. Biochem Biophys Res Commun 353: $1-5,2007$.

66. Cheng TC, Hong C, Akey IV, Yuan S and Akey CW: A near aomic structure of the active human apoptosome. Elife 4: e17755, 2016.

67. Peisley A, Wu B, Xu H, Chen ZJ and Hur S: Structural basis for ubiquitin-mediated antiviral signal activation by RIG-I. Nature 509: 110-114, 2014.

68. Siegel RM, Martin DA, Zheng L, Ng SY, Bertin J, Cohen J and Lenardo MJ: Death-effector filaments: Novel cytoplasmic structures that recruit caspases and trigger apoptosis. J Cell Biol 141: 1243-1253, 1998.

69. Li Y, Fu TM, Lu A, Witt K, Ruan J, Shen C and Wu H: Cryo-EM structures of ASC and NLRC4 CARD filaments reveal a unified mechanism of nucleation and activation of caspase-1. Proc Natl Acad Sci USA 115: 10845-10852, 2018.

70. Lu A, Li Y, Schmidt FI, Yin Q, Chen S, Fu TM, Tong AB, Ploegh HL, Mao Y and Wu H: Molecular basis of caspase-1 polymerization and its inhibition by a new capping mechanism. Nat Struct Mol Biol 23: 416-425, 2016.

This work is licensed under a Creative Commons Attribution-NonCommercial-NoDerivatives 4.0 International (CC BY-NC-ND 4.0) License. 